\title{
Desenvolvimento de espécies de pimentas sobre efeito de doses urina de vaca
}

\section{Development of pepper species on effect of doses of cow urine}

\author{
Lenice Silva ${ }^{1}$; Daniele Lopes de Oliveira ${ }^{2}$; Michele da Silva Santos ${ }^{3}$; Mara Karinne Lopes Veriato Barros ${ }^{* 4}$; \\ Helder Morais Mendes Barros 5
}

Resumo: A crescente demanda do mercado de pimentas tem impulsionado o aumento da área cultivada no Brasil anualmente, no Nordeste brasileiro esse cultivo vem sendo feito não apenas em hortas caseiras para o consumo doméstico, como também em áreas comerciais que abastecem o mercado local e externo. Diante disso objetivou-se verificar os efeitos da utilização da adubação orgânica no cultivo de espécies de pimenta no intuito de possibilitar aos agricultores desenvolverem sua produção utilizando-se de práticas onde produtos alternativos e de baixo custo econômico e ambiental disponíveis em suas propriedades como é o caso da urina de vaca que pode ser utilizados como fertilizante além de possuir outras propriedades favoráveis a este cultivo. Foram usadas as espécies Cambuci, Dedo-de-moça, Bode Amarela e as proporções de urina de vaca utilizadas nas pulverizações foram de 1 a $5 \%$. As variáveis analisadas foram à altura, diâmetro do caule e matéria seca do caule das plantas após a semeadura. A dosagem de urina nas concentrações a partir de $2 \%, 4 \%$ e $5 \%$ obtiveram médias maiores de alturas que nas demais concentrações exercendo maior influência sobre o desenvolvimento das plantas. No diâmetro do caule verificou-se um desenvolvimento maior nas aplicações com concentração de urina aplicada a 4\%, 5\%. E na matéria seca os melhores resultados foram obtidos nas plantas que receberam adubação com urina a 3 a $5 \%$ na maioria das amostras indicando que os melhores efeitos foram atingidos nas maiores concentrações.

Palavras-chave: Cambuci, Dedo-de-moça, Bode Amarela

Abstract: The growing demand of the peppers market has driven the increase of cultivated area in Brazil annually, in the Brazilian Northeast this cultivation has been done not only in home gardens for domestic consumption, as well as in commercial gardens that supply the local and foreign markets. Therefore, it was decided to observe the effects of the use of organic fertilization on the cultivation of peppers in order to enable farmers to develop their production using practices where alternative products and low economic and environmental cost available on their properties as is the case of Cow urine that can be used as fertilizer besides possessing other properties favorable to this crop. The species Cambuci, Finger, Yellow Goat and the proportions of cow urine used in spraying were 1 to $5 \%$.The analyzed variables were the height, stem diameter and dry matter of the stem of the plants after sowing. Urine dosage at concentrations of $2 \%, 4 \%$ and $5 \%$ obtained higher mean values of heights than the other concentrations exerting greater influence on the development of plants. In the diameter of the stem a greater development was observed in the applications with concentration of urine applied to $4 \%, 5 \%$. And in the dry matter the best results were obtained in the plants that received fertilization with 3 to $5 \%$ urine in the majority of the samples indicating that the best effects were reached in the highest concentrations.

Key words: Cambuci, Finger, Yellow Goat

\footnotetext{
*Autor para correspondência

Recebido para publicação em 21/09/2015; aprovado em 23/12/2015

${ }^{1}$ Graduada em História, UEPB e Ciências Agrárias, UFPB, Campina Grande, Paraíba, Brasil .E-mail: lenicesilva1807@gmail.com;;

${ }^{2}$ Doutorando em Eng. Agrícola. CTRN/UFCG, Campina Grande Paraíba, Brasil. E-mail:danielemestre@ hotmail.com;

${ }^{3}$ Mestre em Eng. Agrônomo, Professor a, Unipampa - Universidade Federal do Pampa. Itaqui, Rio Grande do Sul, Brasil. E-mail: michelef1v@ hotmail.com;

${ }^{4}$ Advogada - Especialista em Política e Gestão Pública - Universidade Federal de Campina Grande, Paraíba, Brasil. E-mail: karinnelv@ yahoo.com.br;

${ }^{5}$ Doutor em Eng. Agrícola, CTRN/UFCG, Campina Grande, Paraíba, Brasil. E-mail: hmmbbr@ yahoo.com.br
} 


\section{INTRODUÇÃO}

Plantas condimentares, tais como as pimentas e pimentões do gênero Capsicum, sempre foram usadas pelos índios e civilizações antigas para tornar os alimentos mais agradáveis ao paladar, além de serem utilizadas como conservantes em alimentos, são fontes de antioxidantes naturais como a vitamina $\mathrm{E}$, vitamina $\mathrm{C}$ e carotenoides (REIFSCHNEIDER, 2000). As pimentas também são ricas em capsaicinoides, compostos fenólicos responsáveis pelo sabor pungente ou picante (CARVALHO; BIACHETTI, 2004). Além de suas propriedades nutricionais o sabor é um dos principais atrativos no consumo dessas hortaliças.

Em nível mundial os maiores produtores de pimentas são a Ásia, América Latina, África, Europa e América do Norte. Na Ásia, o agronegócio da pimenta é expressivo, com destaque para Coréia, onde a pimenta constitui o segundo negócio mais importante, após o arroz, e representa $4 \%$ da produção agrícola do país (KWON et al, 2006).

O Brasil é o segundo maior produtor de pimenta no mundo (RISTORI et al., 2002) onde se destaca as regiões Sudeste e Centro-Oeste. O estado de São Paulo é o principal produtor de pimentas e pimentões, e toda produção é destinada ao consumo direto dos frutos como condimentos. No Brasil, indústrias de médio e grande porte que utilizam a pimenta para obtenção de produtos processados estimam que o mercado de pimentas envolve cerca de 80 milhões de reais/ano (RIBEIRO, 2004). O mercado de pimentas pode ser dividido em dois grandes grupos: o consumo in natura, geralmente em pequenas porções, e as formas processadas, que incluem molhos, conservas, flocos desidratados e pó como ingrediente de alimentos processados.

$\mathrm{O}$ mercado para as pimentas sempre foi considerado como secundário no Brasil, o que pode ser resultante do baixo consumo influenciando diretamente no volume de comercialização. Com esse panorama se intensifica a exploração de novos tipos de pimentas e o desenvolvimento de novos produtos, com grande valor agregado, como conservas ornamentais, geleias e outras formas processadas do produto tem apresentado bons resultado (RUFINO; PENTEADO, 2006)

No Nordeste brasileiro, além do cultivo de pimenta em hortas caseiras para o consumo doméstico, existem hortas comerciais que abastecem o mercado local e externo, um dos estados que tem se destacado nessa cultura é no Estado do Ceará com uma área média cultivada no período entre 1998/2000, estimada em 50 hectares. Essa produção é totalmente irrigada e ocorre em 15 municípios do Estado, envolvendo cerca de 140 produtores/ano, nos Vales do Jaguaribe, do Acaraú e do Curu (CRISÓSTOMO, 2006).

No intuito de maximizar a capacidade de fertilidade de solos utiliza-se como alternativa os adubos orgânicos, sendo materiais de origem animal ou vegetal, alguns considerados resíduos ou rejeitos, que têm grande utilização na agricultura orgânica ou ecológica Sua riqueza nutricional promove a elevação da atividade biológica do solo.

Um dos adubos orgânicos utilizado é a urina de vaca que pode ser considerada um subproduto da atividade pecuária, além de amplamente disponível em muitas propriedades rurais. Por ser rica em elementos minerais, considera-se que essa forneça nutrientes e outras substâncias benéficas às plantas a custo reduzido; além disso, seu uso não causa risco à saúde de produtores e consumidores, estando praticamente pronta para uso, bastando apenas acrescentar água (PESAGRO-RIO, 2002).

Resultados positivos da urina de vaca em crescimento de plantas têm sido relatados em pesquisas com culturas de alface e pimentão. Em alface, a aplicação no solo de $20 \mathrm{~mL}$ por planta de solução de urina de vaca na concentração de $0,86 \%$ proporcionou acréscimo de $10,3 \%$ na massa da matéria fresca das plantas (GADELHA et al., 2003). Em pimentão, pulverizações semanais de soluções de urina (0 a 5,0\%) a partir de 15 dias após o transplante até a penúltima colheita proporcionou aumento linear na produção de frutos (OLIVEIRA et al., 2003).

No intuito de obter melhores resultados no desenvolvimento desse cultivo de pimenta objetivou-se avaliar a influência da adubação orgânica feita com aplicação de urina de vaca em dosagens por via foliar, no cultivo de pimenteiras das variedades Cambuci (Capsicum baccatun var. pendulum), Dedo-de-moça (Capsicum baccatum var. pendulum) e Bode amarela (Capsicum chinense) durante todo seu desenvolvimento vegetativo.

\section{MATERIAL E MÉTODOS}

O trabalho foi desenvolvido em casa de vegetação da Escola Técnica Assis Chateaubriand, situada no município de Lagoa Seca localizado na Microrregião Lagoa Seca e na Mesorregião Agreste Paraibano. Sua área é de $109 \mathrm{~km}^{2}$ representando $0.1937 \%$ do Estado, $0.007 \%$ da Região e $0.0013 \%$ de todo o território brasileiro. A sede do município tem uma altitude aproximada de 634 metros distando 109,4 $\mathrm{Km}$ da capital. O acesso é feito, a partir de João Pessoa, pelas rodovias BR 230/BR 104 no período de 02/06 a 04/122010.

Para realização do experimento foram utilizadas sementes de três variedades de pimenta Cambuci ( $C$. baccatun var. pendulum), Dedo-de-moça (Capsicum baccatum var. pendulum) e Bode Amarela (Capsicum chinense), cuja as sementes foram adquiridas em loja de produtos agrícolas. Para montagem do experimento as sementes foram colocadas inicialmente em copos descartáveis embebidas em urina de vaca pura por um período de $24 \mathrm{~h}$ (vinte e quatro horas), durante o processo de embebição, as sementes passaram pelas fases preparatórias essenciais à germinação (FANTI; PEREZ, 2003).

Segundo Popinigis (1985), a germinação do ponto de vista fisiológico, engloba quatro fases a embebição de água, o alongamento das células, a divisão celular e a diferenciação das células em tecidos. Os efeitos provocados pela embebição fazem com que as sementes atinjam um determinado grau de umidade ativando o sistema metabólico que esta relacionado com o processo pré-germinativo e com a elevação da respiração das células embrionárias do cultivar. Após o processo de embebição as sementes foram colocadas em sacos de polietileno de cor preta com dimensões de $15 \times 25 \mathrm{~cm}$ para germinação preenchida com terra vegetal misturado a esterco bovino em uma proporção de 5:1. As plântulas foram transferidas para baldes de PVC com dimensões de 22,3×25 $\mathrm{cm}$ onde foram cultivadas.

A urina foi coletada em vacas leiteiras mestiças, criadas em sistema de semi-confinamento e alimentadas com capim elefante, farelo de soja, mandioca (parte aérea e raiz) e torta de algodão. Antes da aplicação, a urina passou por um período de repouso durante três dias em recipiente plástico com tampa, conforme metodologia propostas por EMATER- 
CE (2000). A pulverização da urina de vaca nas plantas foi feita segundo metodologia proposta por (OLIVEIRA et al 2008) duas vezes por semana.

Durante todo o cultivo das pimenteiras foram realizadas pulverizações periódicas com urina de vaca dissolvida em água em diferentes proporções constituindo 5 (cinco) diferentes tratamentos. As proporções utilizadas nas pulverizações foram para cada $500 \mathrm{ml}$ de água adicionados respectivamente $5 \mathrm{ml}(1 \%), 10 \mathrm{ml}(2 \%), 15 \mathrm{ml} \mathrm{(3 \% ),} 20 \mathrm{ml}$ $(4 \%)$ e $25 \mathrm{ml}(5 \%)$ de urina de vaca. O pulverizador utilizado nesse processo foi o manual e as pulverizações eram realizadas duas vezes por semana tendo inicio a partir do segundo dia em que as pimentas haviam sido transferidas para os recipientes definitivos, nesse caso os baldes.

Empregou-se um esquema fatorial disposto em um delineamento inteiramente casualizado, composto por dois fatores (variedades e dosagens de urina de vaca), perfazendose um total de quinze tratamentos e utilizando-se cinco repetições. Os tratamentos foram assim delineados por pulverizações com urina de vaca de 1 a $5 \%$ na variedade de pimenta Cambuci; perfazendo os tratamentos de 1 a 5 , pulverizações com urina de vaca de 1 a $5 \%$ na variedade de pimenta Dedo de Moça; perfazendo os tratamentos de 6 a 10 e pulverizações com urina de vaca de 1 a $5 \%$ na variedade de pimenta Bode Amarela; perfazendo os tratamentos de 11 a 15.

As variáveis analisadas foram à altura, diâmetro do caule e matéria seca do caule das plantas após a semeadura. Para a altura caulinar utilizou-se uma régua milimétrica, observando-se desde o comprimento da haste principal das plantas até o local de inserção do ultimo par de folhas e/ou último ramo adjacente. Para o diâmetro das plantas (determinado a uma altura de um centímetro na base do caule) foi utilizado um paquímetro. As medições foram feitas no final do experimento. Para determinação da massa seca caulinar de cada planta foi cortada rente ao solo e, em seguida, foram separadas das folhas, posteriormente foram acondicionadas em sacos de papel devidamente identificados e postos para secar em estufa de circulação forçada de ar, mantida na temperatura de $65^{\circ} \mathrm{C}$ até obtenção de massa constante.

\section{RESULTADOS E DISCUSSÃO}

A aplicação de urina de vaca em diferentes dosagens para cada variedade de pimenteira apresentou distintos resultados no comportamento vegetativo das espécies estudadas.

Para avaliação do desenvolvimento da altura de planta foram feitos gráficos para cada espécie cultivada no intuito de observar a média de desenvolvimento por cultivar, vale salientar que as plantas foram expostas a aplicações de urina de vaca a 1 a $5 \%$.

$\mathrm{Na}$ avaliação da altura de planta da variedade Cambuci (C. baccatun var. pendulum) apresentada no Figura 1 as plantas que foram pulverizadas com dosagens de urina de vaca pura em porcentagens $1 \%$ e $2 \%$, obtiveram melhores resultados chegando a alturas que variaram a cima de $1,10 \mathrm{~m}$ e 1,20 $\mathrm{m}$ e a que menos influenciou foi a pulverizada com a dosagem $5 \%$.
Figura 1. Altura de planta $(\mathrm{cm})$ variedade Cambuci $(C$. baccatun var. pendulum) com seus respectivos tratamentos em função da aplicação de diferentes concentrações de urina de vaca

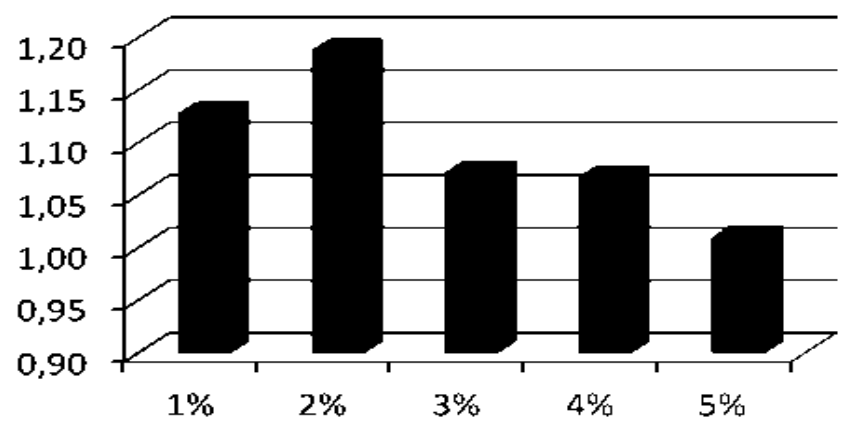

$\mathrm{Na}$ Figura 2 a avaliação da altura de planta da variedade Dedo-de-moça (Capsicum baccatum var. pendulum) observou os melhores resultados com aplicações de urina de vaca pura a $4 \%$, quando as plantas alcançaram alturas a cima de $1 \mathrm{~m}$ que é a altura média desse cultivar. Já nas demais aplicações os resultados forma inferiores a $1 \mathrm{~m}$ o que significa dizer que as plantas tiveram desenvolvimento à baixo da média.

Figura 2. Altura de planta $(\mathrm{cm})$ variedade Dedo-de-moça (Capsicum baccatum var. pendulum) com seus respectivos tratamentos em função da aplicação de diferentes concentrações de urina de vaca.

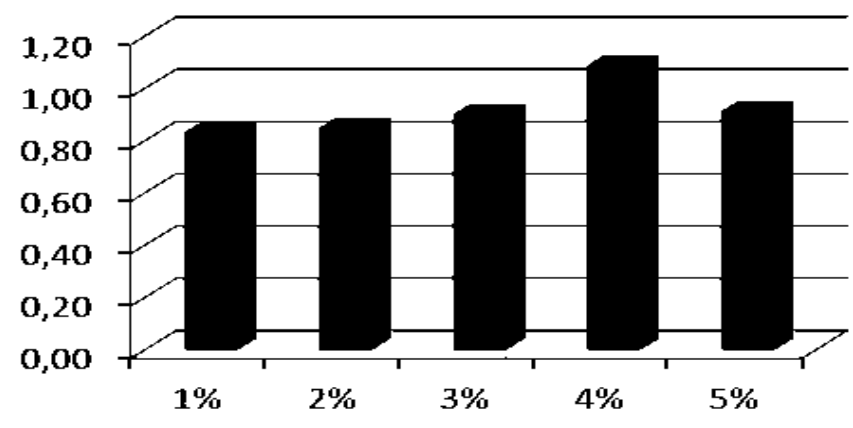

Para a altura de planta da variedade Bode amarela (Capsicum chinense) (Figura 3), as plantas apresentaram melhor desenvolvimento as que receberam pulverizações com urina de vaca a $2 \%$, seguido de $5 \%$ e $1 \%$ atingindo alturas que variaram entre $70 \mathrm{~cm}$ e $80 \mathrm{~cm}$, valores que estão dentro da média de crescimento dessa cultivar que varia entre $70 \mathrm{~cm}$ e $1 \mathrm{~m}$.

Figura 3. Altura de planta $(\mathrm{cm})$ Bode amarela (Capsicum chinense) com seus respectivos tratamentos em função da aplicação de diferentes concentrações de urina de vaca.

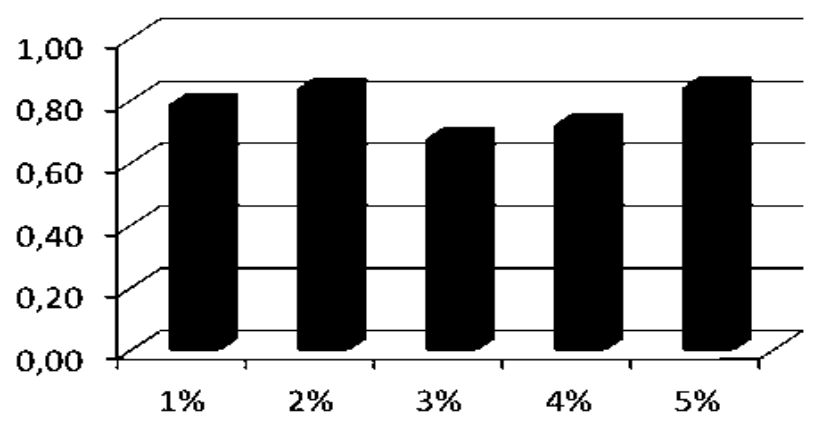


Cesar, et al., (2007) estudando o efeito estimulante da urina de vaca sobre o crescimento de mudas de pepinos, cultivadas, sob manejo orgânico observou que a aplicação da urina de vaca sob a forma de pulverização promoveu estimulo ao desenvolvimento de mudas de pepino. Concordando com os dados de Butrinowski et al. (2013) que encontrou resultados crescentes para a altura da planta de acordo com o implemento de níveis de irrigação em mudas de eucalipto.

$\mathrm{Na}$ variedade Cambuci ( $C$. baccatun var. pendulum), a avaliação de diâmetro do caule (Figura 4)os melhores resultados foram obtidos nas aplicações de urina nas respectivas porcentagens $4 \%, 5 \%$ e $1 \%$ atingindo um diâmetro médio a cima de $1 \mathrm{~cm}$.

Figura 4. Diâmetro de caule $(\mathrm{cm})$ variedade Cambuci $(C$. baccatun var. pendulum) com seus respectivos tratamentos em função da aplicação de diferentes concentrações de urina de vaca.

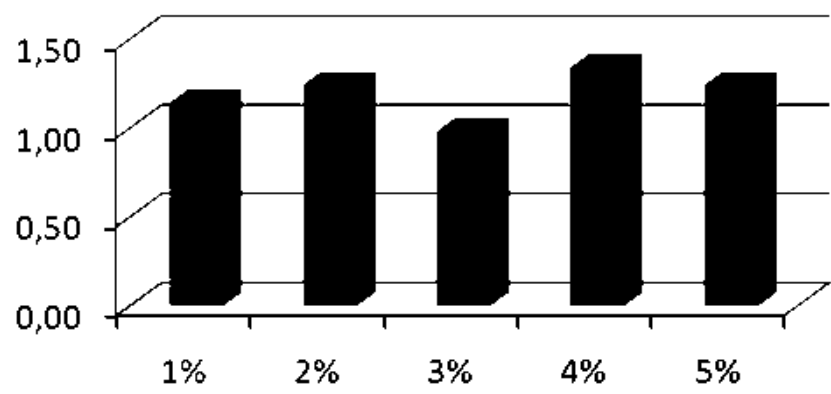

Para a cultivar Dedo-de-moça (Capsicum baccatum var. pendulum) (Figura 5) verificou-se um maior desenvolvimento nas aplicações feitas com urina de vaca a $4 \%$ e $5 \%$, fazendo com que as pimentas atingissem uma média entre $1 \mathrm{~mm}$ e $1,10 \mathrm{~mm}$ de diâmetro de caule.

Figura 5. Diâmetro de caule (cm) variedade Dedo-de-moça (Capsicum baccatum var. pendulum) com seus respectivos tratamentos em função da aplicação de diferentes concentrações de urina de vaca.

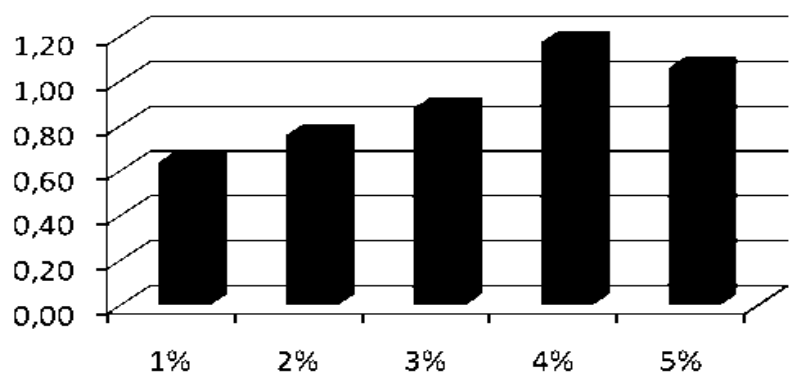

Na avaliação do diâmetro do caule da variedade Bode amarela (Capsicum chinense), que os melhores resultados foram alcançados com aplicações a $4 \%, 5 \%$ e $1 \%$, percebendo-se uma variação de diâmetro entre $1 \mathrm{~cm}$ e 1,50 $\mathrm{cm}$.
Figura 6. Diâmetro de caule $(\mathrm{cm})$ na variedade Bode amarela (Capsicum chinense) com seus respectivos tratamentos em função da aplicação de diferentes concentrações de urina de vaca

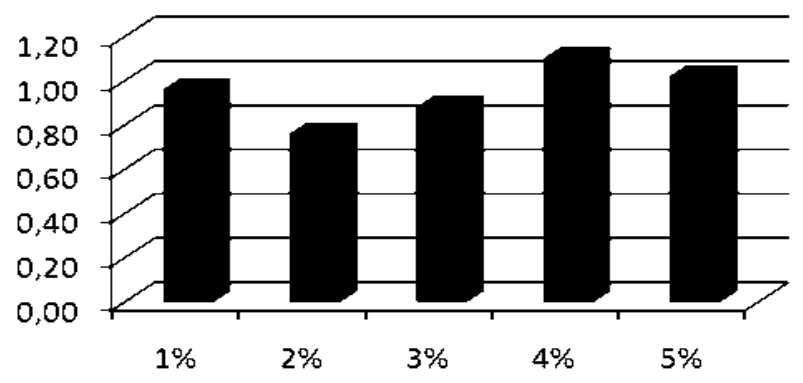

Contrariando os resultados encontrados por Pereira et al. (2010) estudando o efeito da urina de vaca no cultivo do alface e constatou que não houve influência no diâmetro do caule desta planta.

Já na variedade Cambuci (Capiscum baccatun L. var. pendulum)a avaliação da produção de matéria seca apresentou melhor desenvolvimento com as aplicações de urina a $2 \%$ e $4 \%$, seguidas pelas aplicações a 5\%, $1 \%$ diferenciando-se do desenvolvimento apresentado pelas demais variedades que obtiveram melhor resultado a medida que aumentava a porcentagem de urina aplicada.

Figura 7. Produção de matéria seca do caule (g) da variedade Cambuci (Capiscum baccatun L.var. pendulum), com seus respectivos tratamentos em função da aplicação de diferentes concentrações de urina de vaca.

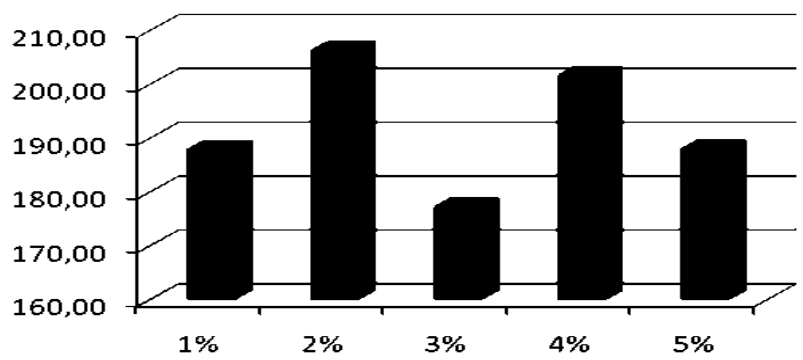

$\mathrm{Na}$ avaliação de matéria seca do caule da variedade Dedo de Moça (Capsicum baccatum var. pendulum) apresentada na Figura 8, os melhores resultados também foram apresentados nas porcentagens de 5\%, 4\%, 3\% e 1\%, nessa variedade a produção de matéria seca variou entre $100 \mathrm{~g}$ e $140 \mathrm{~g}$.

Figura 8. Produção de matéria seca do caule (g) da variedade Dedo de Moça (Capsicum baccatum var. pendulum), com seus respectivos tratamentos em função da aplicação de diferentes concentrações de urina de vaca.

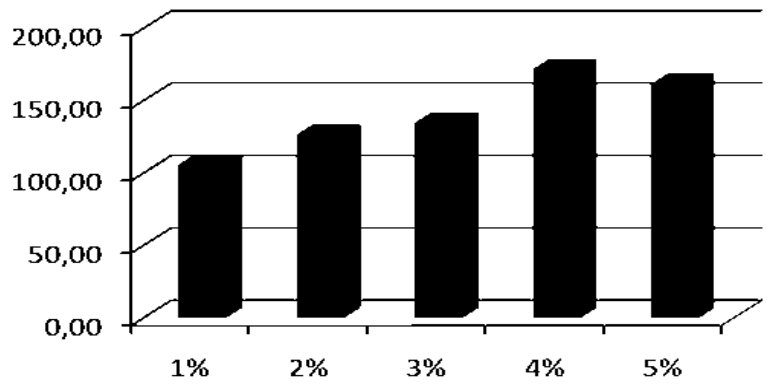


De acordo com o Figura 9 à avaliação da produção de matéria seca do caule da pimenteira de variedade Bode Amarela (Capsicum chinense), o melhor desenvolvimento foi apresentado no tratamento onde a concentração de urina de vaca foi disponibilizada a 5\% seguido pela aplicação feita a $4 \%$,mostrando que nessa variedade a aplicação da urina em dosagens maiores proporcionou um maior desenvolvimento de matéria seca do caule, não podemos deixar de perceber o desenvolvimento apresentado nas aplicações a $3 \%$ e $1 \%$ que também foram significativas quanto a aplicação a $2 \%$ a queda de produção pode ser justificada pela influência dos ventos no momento da aplicação da urina por via foliar.

Figura 9. Produção de matéria seca do caule (g) da variedade Bode Amarela (Capsicum chinense), com seus respectivos tratamentos em função da aplicação de diferentes concentrações de urina de vaca.

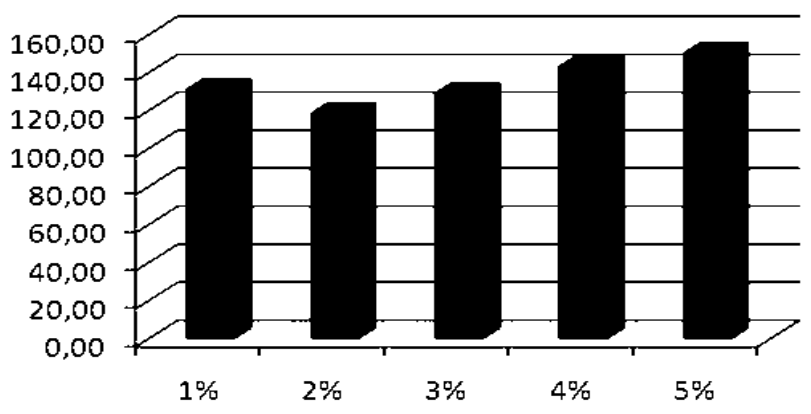

\section{CONCLUSÕES}

A dosagem de urina nas concentrações a partir de $2 \%$, $4 \%$ e $5 \%$ obtiveram médias maiores de alturas que nas demais concentrações exercendo maior influência sobre o desenvolvimento das plantas. O diâmetro do caule obteve desenvolvimento maior nas aplicações com concentração de urina aplicada a $4 \%, 5 \%$.

Quanto à avaliação de produção de matéria seca os melhores resultados foram obtidos nas plantas que receberam adubação com urina a 3 a 5\% na maioria das amostras indicando que os melhores efeitos foram atingidos nas maiores concentrações no entanto na variedade Cambuci (Capiscum baccatun L.var. pendulum), nas amostras em que as pulverizações foram feitas a $2 \%$ e $4 \%$ apresentaram melhor desenvolvimento saindo do padrão das demais variedades esse fato pode ter sido gerado palas condições do vento no momento da pulverização das demais variedades e seus respectivos tratamentos.

Diante do que foi observado e mesmo havendo uma variação nos valores obtidos a utilização da urina de vaca mostrou-se eficiente para suprir as necessidades nutricionais da pimenteira proporcionando o bom desenvolvimento nos três aspectos avaliados: altura, diâmetro do caule e matéria seca do caule.

Mostrando que a aplicação de urina de vaca em variadas concentrações pode ser utilizada na fertirrigação da espécie (capiscum), pois se constitui num recurso acessível ao agricultor não só pelo baixo custo, como também pelo fácil manejo além de não causar dependência de insumos externos que requerem maiores investimentos por parte dos agricultores.

\section{REFERÊNCIAS}

BUTRINOWSKI, R. T. BUTRINOWSKI, I. T. SANTOS, E. L. PICOLlOTO, R. A. SANTOS, R. F. Disponibilidade hídrica no desenvolvimento inicial de mudas de Eucalyptus grandes em ambiente protegido. Acta Iguazu, Cascavel, v.2. n. 3, p. 84-93, 2013

CARVALHO, S. I. C.; BIANCHETTI, L. B. Sistema de produção de pimentas. 2004. Disponível em: <http://www.cnph.embrapa.br/sistprod/pimenta/botanica.htm >. Acesso em: 09 jan. 2013.

CESAR, M. N. Z.; PAULA, P. D. DE; POLIDORO, J. C.; RIBEIRO, R. L. D.; PADOVAN, M. P.; Efeito estimulante da urina de vaca sobre o crescimento de mudas de pepino, cultivadas sob manejo orgânico. Ensaios e Ciência: Ciências Biológicas, Agrárias e da Saúde, vol. 11, núm. 1, abril, 2007, pp. 67-71 Universidade Anhanguera, Campo Grande, Brasil

CRISÓSTOMO, J. R.. Cultivo de pimenta tabasco no Ceará/ (editor técnico) - Fortaleza : Embrapa Agroindústria Tropical, 2006. 40 p. (Embrapa Agroindústria Tropical. Sistema de Produção, 3).

EMATER-CE. Urina de vaca: adubo e defensivo natural para o solo e plantas. Fortaleza: SRD, 2000. 3p. (Boletim Informativo).

FANTI, S. C.; PEREZ, S. C. J. G. A. Influência do sombreamento artificial e da adubação química na produção de mudas de Adenanthera pavonina L. Ciência Florestal, Santa Maria, v. 13, n. 1, p. 49-56, 2003.

GADELHA RSS, CELESTINO RCA, SHIMOYA A (2003) Efeito da utilização de urina de vaca na produção da alface. Pesquisa Agropecuária \& Desenvolvimento Sustentável, $1: 179-182$.

KWON, T. R.; PAE, D. H.; SHIN, Y. A.; OH, D. G.; LEE, J. M. Capsicum peppers: a vital crop for Korea. Horticultural Science News. v.46, n. 1, p. 16-19. 2006.

OLIVEIRA, M.L.; BACCARO, F.B.; BRAGA-NETO, R.; MAGNUSSON,.W.E. 2008. Reserva Ducke: a biodiversidade amazônica através de uma grade. Manaus, Brasil. 166 pp.

OLIVEIRA A. P.; PAES R. A.; SOUZA A. P.; DORNELAS C. S. M. Rendimento de pimentão adubado com urina de vaca e NPK. In: 43 Congresso Brasileiro de Olericultura, Recife. Resumos, SOB. 2003.CD-ROM.

PEREIRA, P. M; CARVALHO, V. N.; BASTOS, A. L.; NASCIMENTO JÚNIOR, N. A. do. Efeito da urina de vaca no cultivo da alface. CONNEPI, 1, 2010, Alagos. Anais... Alagoas: IFAL, 2010.

POPINIGIS, F. Fisiologia da semente. Brasília, 2. ed., 1985. $298 \mathrm{p}$.

PESAGRO-RIO Urina de vaca: alternativa eficiente e barata.Rio de Janeiro, Documentos, n. 96. 8p. 2002. 
REIFSCHNEIDER, F. J. B. (Org.) Capsicum: pimentas e pimentões no Brasil. Brasília: Embrapa Comunicação para Transferência de Tecnologia/Embrapa Hortaliças, 2000.

RISTORI, C. A.; PEREIRA, M. A. S.; GELLI, D. S. O efeito da pimenta do reino moída frente a contaminação in vitro com Salminella Rubslaw. Rev. Inst. Adolfo Lutz, v. 62, n. 2, p. 131-133, 2002.

RIBEIRO, C. S. da C. Apresentação do Encontro Nacional do agronegócio pimentas (Capsicum spp.). In: ENCONTRO NACIONAL DO AGRONEGÓCIO, I, 2004. Brasília. Anais... Brasilia: CNPH, 2004. 1 CD-ROM.

RUFINO, J. L. S.; PENTEADO, D. C. S. Importância econômica, perspectivas e potencialidades do mercado para pimenta. Informe Agropecuário, v. 27, n. 235, p.7-15, 2006. 